\title{
Empirical Mode Decomposition as a Filter Bank
}

\author{
Patrick Flandrin, Fellow, IEEE, Gabriel Rilling and Paulo Gonçalvès
}

\begin{abstract}
Empirical Mode Decomposition (EMD) has recently been pioneered by N.E. Huang et al. for adaptively representing nonstationary signals as sums of zero-mean AM-FM components [2]. In order to better understand the way EMD behaves in stochastic situations involving broadband noise, we report here on numerical experiments based on fractional Gaussian noise. In such a case, it turns out that EMD acts essentially as a dyadic filter bank resembling those involved in wavelet decompositions. It is also pointed out that the hierarchy of the extracted modes may be similarly exploited for getting access to the Hurst exponent.
\end{abstract}

Index Terms-Empirical Mode Decomposition, filter banks, wavelets, fractional Gaussian noise.

\section{EMD BASICS}

$\mathbf{T}$ HE starting point of the Empirical Mode Decomposition (EMD) is to consider signals at the level of their local oscillations. Looking at the evolution of a signal $x(t)$ between two consecutive local extrema (say, two minima occurring at times $t_{-}$and $t_{+}$), we can heuristically define a (local) highfrequency part $\left\{d(t), t_{-} \leq t \leq t_{+}\right\}$. Also called detail, $d(t)$ corresponds to the oscillation terminating at the two minima and passing through the maximum which necessarily exists in between them. For the picture to be complete, we also identify the corresponding (local) low-frequency part $m(t)$, or local trend, so that we have $x(t)=m(t)+d(t)$ for $t_{-} \leq t \leq$ $t_{+}$. Assuming that this is done in some proper way for all the oscillations composing the entire signal, we get what is referred to as an Intrinsic Mode Function (IMF) as well as a residual consisting of all local trends. The procedure can then be applied to this residual, considered as a new signal to decompose, and successive constitutive components of a signal can therefore be iteratively extracted, the only definition of such a so-extracted "component" being that it is locally (i.e., at the scale of one single oscillation) in the highest frequency band.

Given a signal $x(t)$, the effective algorithm of EMD can be summarized as follows [2]:

1) identify all extrema of $x(t)$

2) interpolate between minima (resp. maxima), ending up with some "envelope" $e_{\min }(t)$ (resp. $e_{\max }(t)$ )

3) compute the average $m(t)=\left(e_{\min }(t)+e_{\max }(t)\right) / 2$

4) extract the detail $d(t)=x(t)-m(t)$

5) iterate on the residual $m(t)$

In practice, the above procedure has to be refined by a sifting process which amounts to first iterating steps 1 to 4 upon

Manuscript received November 6, 2002; revised February 18, 2003.

P. Flandrin and G. Rilling are with the Laboratoire de Physique (UMR 5672 CNRS), École Normale Supérieure de Lyon, 46 allée d'Italie, 69364 Lyon Cedex 07 France (email : flandrin,grilling@ens-lyon.fr).

P. Gonçalvès is with Projet IS2, INRIA Rhone-Alpes, 655 avenue de l'Europe, 38334 Saint Ismier Cedex France (email: Paulo.Goncalves@inria.fr). the detail signal $d(t)$, until this latter can be considered as zero-mean according to some stopping criterion. Once this is achieved, the detail is considered as the effective IMF, the corresponding residual is computed and step 5 applies.

By construction, the number of extrema is decreased when going from one residual to the next (thus guaranteeing that the complete decomposition is achieved in a finite number of steps), and the corresponding spectral supports are expected to decrease accordingly. While modes and residuals can intuitively be given a "spectral" interpretation, it is worth stressing the fact that, in the general case, their high vs. low frequency discrimination applies only locally and corresponds by no way to a pre-determined subband filtering (as, e.g., in a wavelet transform). Selection of modes rather corresponds to an automatic and adaptive (signal-dependent) time-variant filtering.

\section{EMD ANALYSIS OF FRACTIONAL GAUSSIAN NOISE}

For a given signal $x(t)$, EMD ends up with a representation of the form:

$$
x(t)=m_{K}(t)+\sum_{k=1}^{K} d_{k}(t),
$$

where $m_{K}(t)$ stands for a residual "trend" and the "modes" $\left\{d_{k}(t), k=1, \ldots K\right\}$ are constrained to be zero-mean AMFM waveforms. As such, EMD proves especially efficient in those deterministic situations which precisely enter the framework of "sinusoidal models" (at large) [2]. At the notable exception of a recent study [5], much less attention has been paid to more realistic situations involving noise, and little is known indeed on the decomposition achieved by EMD when the analyzed signal is only the realization of some stochastic process. We propose in this letter to address this issue by resorting to fractional Gaussian noise as a versatile model for broadband noise.

\section{A. Fractional Gaussian noise}

Let us recall that fractional Gaussian noise (fGn) is defined as the increment process of fractional Brownian motion [3]. In discrete-time, fGn corresponds to a time series $\left\{y_{H}[n], n=\right.$ $\ldots,-1,0,1, \ldots\}$ indexed by a real-valued parameter $0<$ $H<1$ (its Hurst exponent), and such that its autocorrelation sequence $r_{x_{H}}[k]:=\mathbb{E}\left\{y_{H}[n] y_{H}[n+k]\right\}$ reads:

$$
r_{x_{H}}[k]=\frac{\sigma^{2}}{2}\left(|k-1|^{2 H}-2|k|^{2 H}+|k+1|^{2 H}\right) .
$$

As is well-known, the special case $H=1 / 2$ reduces to white noise, whereas other values induce non-zero correlations, either negative when $0<H<1 / 2$ or positive when $1 / 2<H<1$ (long-range dependence). 

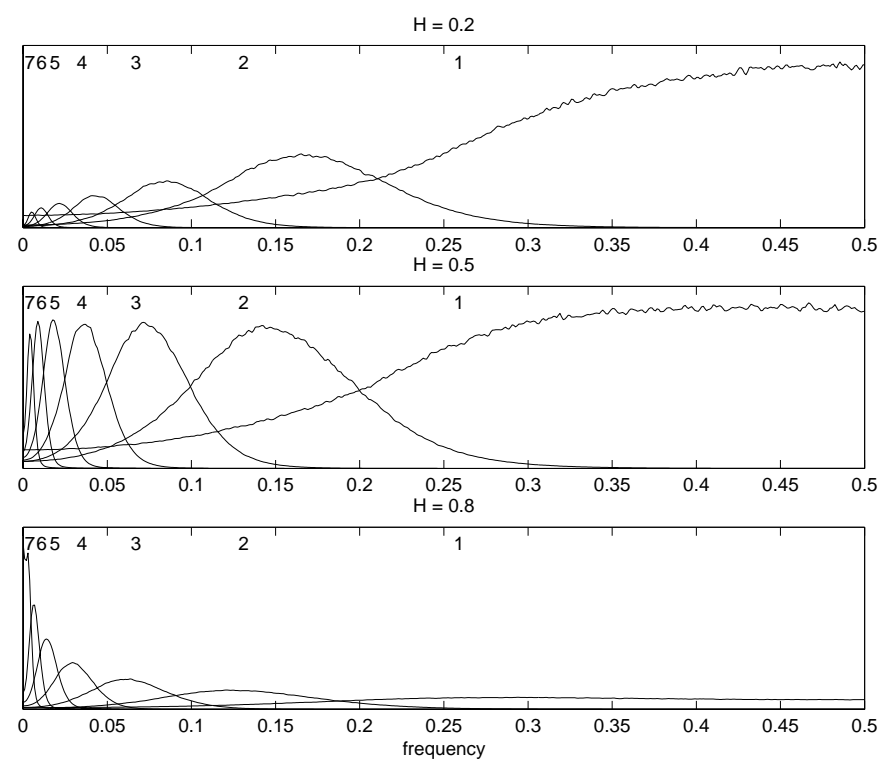

Fig. 1. EMD equivalent filters - In the case of fractional Gaussian noise, EMD can be interpreted as a filter bank of overlapping band-pass filters for modes of indices $k \geq 2$, the mode \#1 corresponding essentially to a half-band high-pass filter. For each value of the Hurst exponent ( $H=0.2,0.5$ and 0.8$)$, 5000 independent time series of 512 points each have been generated, and the average spectra of the 7 first IMFs are plotted as a function of normalized frequency.

We will here report on extensive simulations that have been carried out on such processes, with $H$ varying from 0.1 to 0.9 . The data length has been typically set to $N=512$ and, for each value of $H, 5000$ independent sample paths of fGn have been generated via the Wood and Chan algorithm [4]. Details on the effective implementation of the EMD algorithm will not be given here, but the Matlab codes used for the simulations are available [7].

\section{B. Equivalent filters}

A first analysis of the fGn time series consists in estimating a power spectrum for each mode of the decomposition. To this end, empirical autocorrelation functions are first computed for each mode of each realization, prior being ensemble averaged over all realizations, tapered and Fourier transformed. This results, mode by mode, in a frequency profile that can be interpreted as the frequency output of some equivalent filter. As evidenced in Figure 1, the collection of all such filters tend to organize in a filter bank structure which is reminiscent of what is classically observed in wavelet decompositions in similar situations [1]. Indeed, while the filter associated to the mode \#1 is essentially high-pass (although it contains a nonnegligible low-pass part in the lower half-band), the modes of higher indices are characterized by a set of overlapping bandpass filters. Moreover, each mode of index $(k+1), k \geq 2$, occupies a frequency domain which is roughly the upper halfband of that of the previous residual of index $k$. It is worth to point out that similar results have been obtained independently by $\mathrm{Wu}$ and Huang [5] in the case of white noise (corresponding here to $H=1 / 2$ ).

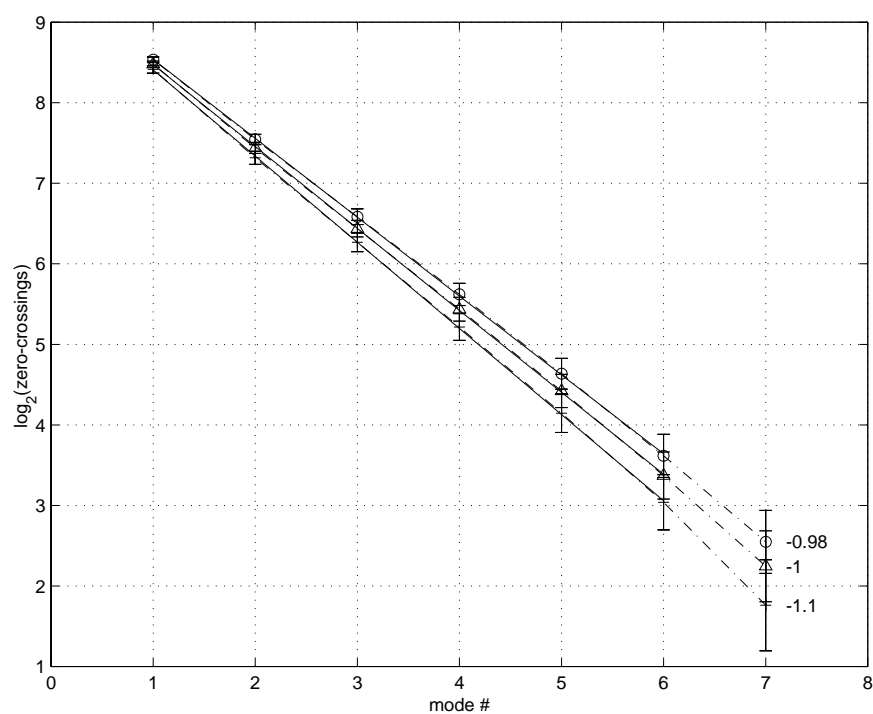

Fig. 2. IMF zero-crossings - The (base 2) logarithm of the average number of zero-crossings is plotted in dashed-dotted lines as a function of the IMF number, for 3 different values of the Hurst exponent ( $H=0.2$ (crosses), 0.5 (circles) and 0.8 (squares)). We observe in each case a straight line whose slope (estimated by a linear fit over the 6 first modes, superimposed full lines) is very close to -1 , indicating an almost dyadic decrease across modes.

\section{Filter bank structure}

The qualitative appreciation given above about the equivalent filter bank structure of EMD can be quantified further as follows. By construction, each IMF is a zero-mean waveform whose number of zero-crossings differs at most by one from the number of its extrema. The number of these zero-crossings is a rough indication of the mean frequency of each mode, and the way this number varies from mode to mode is a further indication of the hierarchical structure of the equivalent filter bank. As evidenced in Figure 2, the number of zero-crossings $z_{H}[k]$ is a decreasing exponential function of the mode number $k$ :

$$
z_{H}[k] \propto \rho_{H}^{-k}
$$

with $\rho_{H}$ very close to 2 .

Using this result, we can further check for a possible selfsimilarity in the filter banks of Figure 1. Denoting by $\mathcal{S}_{k}(f)$ the power spectrum of the $k$-th IMF $d_{k}(t)$, self-similarity of the band-pass filters would mean that

$$
\mathcal{S}_{k^{\prime}}(f)=\rho_{H}^{\alpha\left(k^{\prime}-k\right)} \mathcal{S}_{k}\left(\rho_{H}^{k^{\prime}-k} f\right)
$$

for some $\alpha$ and any $k^{\prime}>k \geq 2$, and hence that the power spectra of all IMF's could be collapsed onto a single curve, when properly renormalized. Such a collapse via renormalization can be observed on Figure 3, obtained with the specific choice $\alpha=2 H-1$. Even if some low frequency discrepancies can be observed (especially when $H<1 / 2$ ), these diagrams support the claim that, in a first approximation (and in agreement with the findings reported in [5] for white noise), EMD acts on fGn as a dyadic filter bank of constant- $Q$ band-pass filters. 
$\mathrm{H}=0.2$
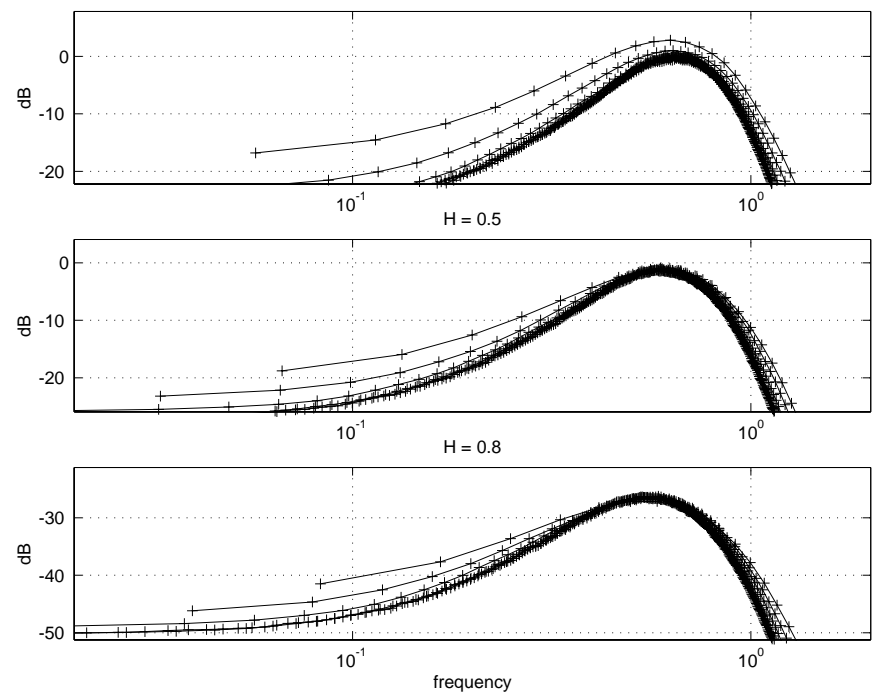

Fig. 3. EMD filter bank renormalization - When renormalized according to the r.h.s. of (4) with $\alpha=2 H-1$ and $\rho_{H}$ as obtained from the slopes of Figure 2, the band-pass frequency profiles of Figure 1 (i.e., the spectra corresponding to modes 2 to 7 , plotted here in log-log coordinates) almost collapse onto a single curve. This supports the claim that, in a first approximation, the equivalent filter bank structure of EMD is dyadic with constant- $Q$ band-pass filters.

\section{Estimation of $H$}

Using the filter bank structure described above, it becomes possible to get access to the Hurst exponent $H$ via the variance progression across IMF's. In fact, assuming that the renormalization equation (4) is satisfied by the considered IMF's, it immediately follows that their variance should be such that:

$$
\operatorname{var} d_{k}(t) \propto \rho_{H}^{(\alpha-1) k} .
$$

Examples of log-variance progressions are plotted in the top graph of Figure 4, with the corresponding linear fits and $H$ estimates. It turns out that the expected straight lines are obtained for $H=0.5$ and 0.8 but that a significant bending occurs for $H=0.2$.

While only 3 typical values of $H$ are displayed on this Figure for the sake of readability, it appears from more complete experiments within the range $0.1 \leq H \leq 0.9$ that the observed behaviour is quite general and that the linear dependence of the log-variance as a function of the mode index is only a gross approximation when $H<1 / 2$. In other words, a self-similar model for the considered filter bank makes sense mostly for $H \geq 1 / 2$, as expected from the approximated renormalization collapse of Figure 3. This is further supported by the bottom graph of Figure 4 in which we can observe, for the measured slope, a good agreement with the model (5) when $H \geq 1 / 2$ and a clear deviation from it when $H<1 / 2$.

\section{CONCLUSION}

We reported here on first numerical experiments aimed at supporting the claim that, in the case of structured broadband stochastic processes such as fractional Gaussian noise, the built-in adaptivity of EMD makes it behave spontaneously
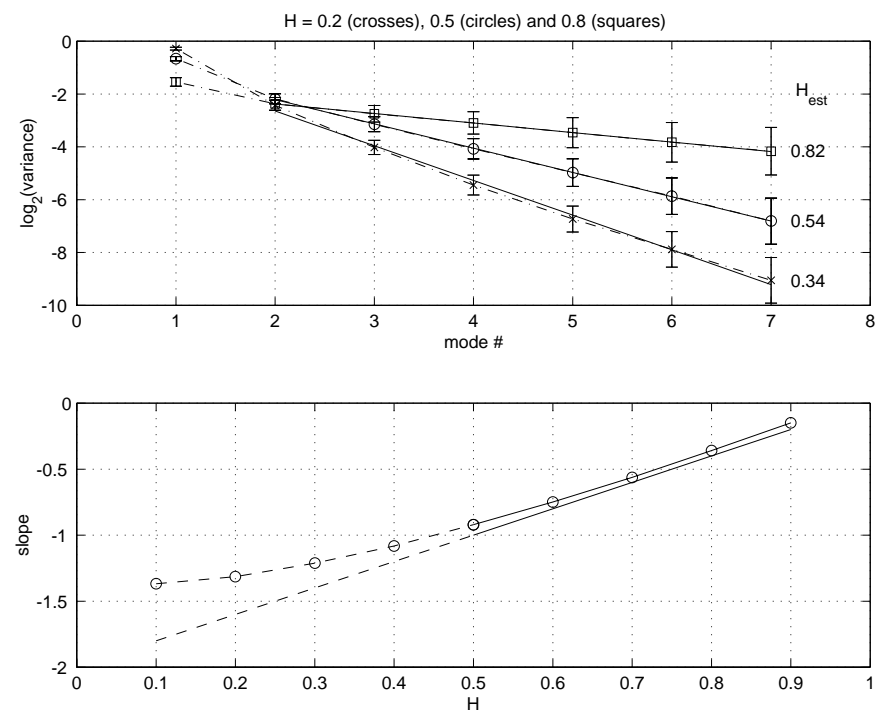

Fig. 4. IMF variance and estimation of the Hurst exponent $H-$ Top: the (base 2) logarithm of the average variance is plotted as a function of the IMF number, for 3 typical values of the Hurst exponent ( $H=0.2$ (crosses), 0.5 (circles) and 0.8 (squares)), with the corresponding linear fits and $H$ estimates. Bottom: when plotted as a function of the Hurst exponent $H$, the IMF log-variance slope $p(H)$ is almost linear when $H \geq 1 / 2$, in accordance with the simplified model $p(H)=2(H-1)$ predicted by self-similarity and the approximation $\rho_{H}=2$ (superimposed straight line).

as a "wavelet-like" filter bank. An interesting by-product of this interpretation is that EMD may offer a new way of analyzing self-similar processes. Thorough comparisons (which are beyond the scope of this letter) with other existing methods are in progress. Let us just mention that benefits very similar to those of wavelet-based methods are obtained when using EMD: in particular, the technique happens to naturally cope with superimposed smooth trends.

From a more general perspective, the results presented here clearly call for theoretical elements which would explain the observed behaviours (e.g., the $H$-dependence of the filter bank structure), a task which is made difficult by the fact that EMD does not admit an analytical definition. The purpose of the present experimental study was to be a contribution aimed at a better understanding of one specific aspect of EMD (the way it decomposes broadband noise), filling somehow the gap between a still non-existing theory and the application of an appealing method to real-world situations (see, e.g., [6]).

\section{ACKNOWLEDGMENT}

Dr N.E. Huang from NASA Goddard Space Flight Center is gratefully acknowledged for having brought to our attention Refs. [5] and [6].

\section{REFERENCES}

[1] P. Abry, P. Flandrin, M. Taqqu, and D. Veitch, "Wavelets for the analysis, estimation, and synthesis of scaling data," in Self-Similar Network Traffic and Performance Evaluation (K. Park and W. Willinger, eds.), pp. 3988 , Wiley, 2000.

[2] N.E. Huang, Z. Shen, S.R. Long, M.L. Wu, H.H. Shih, Q. Zheng, N.C. Yen, C.C. Tung and H.H. Liu, "The empirical mode decomposition and Hilbert spectrum for nonlinear and non-stationary time series analysis," Proc. Roy. Soc. London A, Vol. 454, pp. 903-995, 1998. 
[3] B.B. Mandelbrot and J.W. van Ness, "Fractional Brownian motions, fractional noises and applications," SIAM Rev., Vol. 10, pp. 422-437, 1968.

[4] A.T. Wood and G. Chan, "Simulation of stationary processes in $[0,1]^{d}$," J. Comp. Graph. Stat., Vol. 3, pp. 409-432, 1994.

[5] Z. Wu and N.E. Huang, "A study of the characteristics of white noise using the Empirical Mode Decomposition method," submitted to Proc. Roy. Soc. London A, Dec. 2002.

[6] Z. Wu, E.K. Schneider, Z.Z. Hu and L. Cao, "The impact of global warming on ENSO variability in climate records," COLA Technical Report, CTR 110, Oct. 2001.

[7] http://www.ens-lyon.fr/ flandrin/software.html. 